\title{
Optimizing Specific Design for MPB64 in Mycobacterium tuberculosis as a Rapid Diagnosis Candidate for Pulmonary Tuberculosis
}

\author{
Elizabeth Bahar ${ }^{1}$, Ellyza Nasrul ${ }^{2}$, Netti Suharti ${ }^{3}$, Djong Hon Tjong ${ }^{4}$ \\ \{elizabethbahar@med.unand.ac.id ${ }^{1}$, ellyza_nasrul@yahoo.com² ${ }^{2}$,nettisuharti@med.unand.ac.id ${ }^{3}$, \\ djonghontjong@fmipa.unand.ac.id $\left.{ }^{4}\right\}$ \\ Department of Microbiology, Faculty of Medicine, Universitas Andalas ${ }^{1}$ \\ Department of Clinical Pathology, Faculty of Medicine, Universitas Andalas ${ }^{2}$ \\ Department of Biology, Faculty of Mathematics and Science, Universitas Andalas ${ }^{3}$
}

\begin{abstract}
The existing tuberculosis diagnostic with Ziehl Neelsen staining method is a main pillar in enforcing rapid and accurate diagnosis, which has low sensitivity and specificity. On the other hand, the culture method has become a gold standard, needs a longer time. This study aims to determine the specifics of MPB64 in M. tuberculosis as an alternative to the rapid diagnosis of pulmonary tuberculosis. A molecular-based crosssectional study was conducted on 100 sputum samples of suspected TB patients. We specifically identified the variation and conserved sequence of MPB64 in M. tuberculosis in the polymorphisms. We designed a specific primer for MPB64 in M. tuberculosis conserved gene as an alternative to the rapid and accurate molecular diagnosis of tuberculosis. We found 48 positive and 52 negatives in cultures, meanwhile 35 positive and 65 negative PCR results. In the specific design of MPB64 of M. tuberculosis, we found a sequence with the primer code of F-MPTAE2 and R- MPTAE2, which is 20 bp-length. In conclusion, the specific design of MPB64 M. tuberculosis which is self-designed has potential in PCR method for M. tuberculosis diagnosis.
\end{abstract}

Keywords: Culture, Diagnostic test, Primer design, Tuberculosis.

\section{Introduction}

Tuberculosis (TB) is an infectious disease caused by Mycobacterium tuberculosis. Onethird of the world's population is reported to have been latently infected with the bacterium. The burden of TB in the world has reached 10.4 million with the new cases consisting of 5.9 million men, 3.5 million women, and 1 million children. About 1.8 million people die from TB, consisting of 1.1 million men, 0.5 million women, and 0.2 million children. Moreover, 1.4 million people complicated with TB-HIV. Many TB cases are not cured, especially in developing countries [1]. Moreover, the burden is also triggered by the emergence of a pandemic of Human Immunodeficiency Virus (HIV) [2] and Multi Drugs Resistance (MDR) against antiTB drugs, which leads to the difficulty if case management and high mortality [3], [4].

Indonesia has 254.831.222 inhabitants, which occupies the second position of countries with the most frequent TB cases after India, as there are 1.000.000 new cases and 110.000 deaths annually. Meanwhile, the country also has the highest number of unreported cases (missing cases) as many as 690.000 cases of TB which would have affected the high mortality rate [5]. 
In addition to the high number of the cases, there is also a problem of TB prevention in conducting a proper and correct diagnosis.

It is apparent that a rapid and accurate diagnosis method is needed to optimise prevention, by overcoming the limitations of existing methods. The incomplete map of bacterial characteristics of tuberculosis that circulates throughout the region, including genotypic maps and bacterial resistant patterns against anti-tuberculosis drugs is another problem in the diagnosis in Indonesia, such as M. tuberculosis characteristic data can be used for the development of effective and efficient TB diagnostics to detect the type of $M$. tuberculosis in the entire region. A quick and precise diagnosis as early detection can help to determine treatment and break the chain of transmission.

The development of various methods of molecular biology in the identification has been done to detect $\mathrm{M}$. tuberculosis, such as using polymerase chain reaction (PCR). This molecular technique is considered having a high sensitivity and specificity. The polymerase chain reaction (PCR) is the examination method which principally amplifies in-vitro DNA enzymatically against specific target genes of $M$. tuberculosis strains such as IS6110, GroEL0, Pab, E-SAT6 and MPB64.

M. tuberculosis is known to have 4000 genes built by 4.4 x 106 base pairs (bp) with $40 \%$ of them being expressed into proteins and about 33 different proteins being secreted by these bacteria. One of the most important proteins is Mannose Binding Protein 64 or Mycobacterium Protein Tuberculosis (MPB64 / MPT64) consisting of 660 base pairs found only in $M$. tuberculosis and not present in any other Mycobacterium [6]. The MPB64 gene is a specific sequence of $M$. tuberculosis [7] and is often used for the diagnosis of TB, since false positives are low (10\%) when compared with IS6110 (62\%) [8], [9].

The MPB64 gene is suspected to have a mutation which causes polymorphism in the target gene [10]-[12]. As a result, the use of the MPB64 M. tuberculosis target gene in TB diagnosis often results in varying sensitivity and specificity [13]-[15]. This study was designed to examine the variation of mutations in specific MPB64 M. tuberculosis genes in patients suspected of pulmonary TB, and to identify specifics of MPB64 M. tuberculosis (conserved) genes that have been designed and optimised as candidates for pulmonary tuberculosis diagnostic testing.

\section{Materials and Methods}

A cross-sectional study was conducted on suspected pulmonary tuberculosis patients. We used PCR to detect the target genes MPB64 M. tuberculosis and polymorphism. A total of 100 samples in the form of sputum from TB patients were collected from Regional Pulmonary Hospital of West Sumatera, which is located in Lubuk Alung, Padang Pariaman District.

The sputum collection and work was done in Lubuk Alung Hospital microbiology lab using BSC II (1300 SERIES A). The morning sputum of a suspected TB patient based on chest X-ray was placed in a sputum pot provided. In the microbiology laboratory, we performed DNA isolation on the prepared sputum and TB colonies that grow on LJ medium. The DNA isolation method of sputum used a purple Genomic DNA Mini Kit / 50rx K1820-01 (Invitrogen) and was from colonies using the Boillings method. The PCR process was performed on DNA isolates using the MPB64 with sequence code - F_MPT01-AE 5 '- ACG TGG GAC CAA TG CTG GGT T-3' and R_MPT01-AE 5 '- CAG GCG TGC CAG ATT CAA ATG T -3' which amplified the DNA fragments along $240 \mathrm{bp}$. The PCR product was tested using gel electrophoresis at $1 \%$ 
agarose and visualised using Gel doc. The electrophoresis result showed that the position of the band was in $240 \mathrm{bp}$.

\section{Results}

This research was conducted in two stages. The first phase, the molecular variation of the MPB64 M. tuberculosis was analysed. In the second phase, we specifically designed for MPB64 as the selected diagnostic candidate. The study was conducted on 100 samples with 20 of them used for molecular variation analysis. The characteristic of the respondent in this study was presented in Table 1.

Table 1. Characteristics of Respondent

\begin{tabular}{cl}
\hline Parameters & $\mathrm{n}(\%)$ \\
\hline Gender & \\
$\bullet$ Male & $63(63 \%)$ \\
$\bullet$ Female & $37(37 \%)$ \\
Age (years) & $16(16 \%)$ \\
$\bullet 15-25$ & $17(17 \%)$ \\
$\bullet 26-36$ & $15(16 \%)$ \\
$\bullet 37-47$ & $20(20 \%)$ \\
$\bullet 48-58$ & $25(25 \%)$ \\
$\bullet 59-69$ & $6(6 \%)$ \\
$\bullet>70$ & \\
\hline
\end{tabular}

\subsection{Tuberculosis Test}

The diagnosis was performed based on three TB test methods: staining, culture, and PCR on 100 sputum samples. The PCR test used standard for MPB64 M. tuberculosis with sequence code F_MPT01-AE / R_MPT01-AE, Sequence- F_MPT01-AE 5' - ACG TGG GAC CAA TAC CTG GGT T -3', and Sequence- R_MPT01-AE 5' - CAG GCG TGC CAG ATT CAA ATG T -3 '.

Table 2. Comparison of Sputum Test Culture and PCR Methods

\begin{tabular}{lllllll}
\hline Methods & Positive & \multicolumn{3}{c}{ Negative } & Total \\
\hline & $\mathrm{n}$ & $\%$ & $\mathrm{n}$ & $\%$ & $\mathrm{n}$ & $\%$ \\
\hline Culture & 48 & 48 & 52 & 52 & 100 & 100 \\
PCR & 35 & 35 & 65 & 65 & 100 & 100 \\
\hline
\end{tabular}

Table 2 described the distribution of tuberculosis diagnosis based on culture examination, and PCR showed varied results. A positive sputum examination was obtained from a culture examination as much as $48 \%$. 


\subsection{Molecular Variation Analysis}

The total size of the MPB64 M. tuberculosis gene is $660 \mathrm{bp}$ with the limitation of the sequencing engine causing not all parts of DNA to be in the order of the base. The analysis should use 2 pairs of primer with code (F_MPT01_AE / R_MPT01AE and F_MPT02_AE / R_MPT02_AE, Sequence- F_MPT01_AE / R_MPT01AE), F 5 'ACG TGG GAC CAA TAC CTG GGT T 3', and R 5 'CAG GCG TGC CAG ATT CAA ATG T 3'. Sequence- F_MPT02_AE / R_MPT02_AE, F 5 'TGG TAT GTG GGG GAG GTG ATA3', R 5 'GCG ATG CCC TAT GTT TGT GCG 3'.

The sequences were combined using a DNA star. Chromatogram results were edited using Bioedit, and the sequences obtained were analysed using BLAST and Clustal Omega.

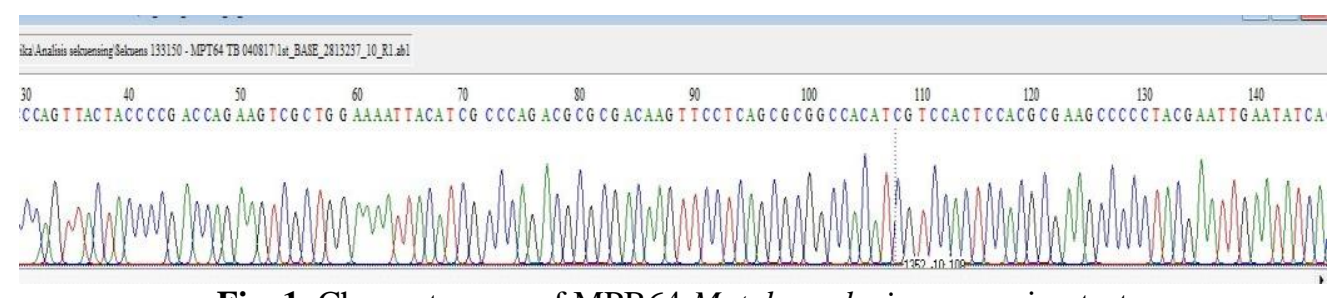

Fig. 1. Chromatogram of MPB64 M. tuberculosis sequencing test

The result of sequencing is presented in a chromatogram with a colourful peak to distinguish the type of nucleotide it represents (Fig. 1). Nucleotide A (Adenine) is green, nucleotide $\mathrm{G}$ (Guanine) is black, nucleotide $\mathrm{C}$ (Cytosine) is blue, and nucleotide T (Timin) is red. The file can be opened using Bioedit offline program.

Table 3. Molecular variation of twenty samples using MPB64 H37Rv

\begin{tabular}{llll}
\hline \multirow{2}{*}{ Sample } & \multicolumn{2}{l}{ Number of molecular variation } & Mutation type \\
\cline { 2 - 3 } & $\mathrm{n}$ & $\%$ & \\
\hline 1 & 2 & 0.3 & Insertion, Deletion \\
2 & 2 & 0.3 & Substitution \\
3 & 2 & 0.3 & Substitution \\
4 & 5 & 0.75 & Insertion, Substitution \\
5 & 1 & 0.15 & Insertion \\
6 & 2 & 0.3 & Insertion, Deletion \\
7 & 1 & 0.15 & Insertion \\
8 & 8 & 1.2 & Deletion, Insertion \\
9 & 1 & 0.15 & Insertion \\
10 & 2 & 0.3 & Insertion, Deletion \\
11 & 1 & 0.15 & Insertion \\
12 & 5 & 0.75 & Insertion, substitution \\
13 & 1 & 0.15 & Insertion \\
14 & 2 & 0.3 & Substitution \\
15 & 1 & 0.15 & Insertion \\
16 & 2 & 0.3 & Substitution \\
17 & 2 & 0.3 & Insertion, Deletion \\
18 & 1 & 0.15 & Insertion \\
19 & 2 & 0.3 & Insertion, Deletion \\
20 & 1 & 0.15 & \\
\hline
\end{tabular}


The molecular analysis of 20 samples showed a very little variation of the MPB64 $M$. tuberculosis gene, (i.e. $0.33 \pm 0.26 \%$ ) with variations spread between $0.15-1.2 \%$. The largest variation was found in sample number $8(1.2 \%)$ followed by samples 4 and $12(0.75 \%)$. The complete results are shown in table 3.

Analysis of the type of mutation shows the three forms of mutation (insertion, deletion, and substitution) found in all isolates, but with different proportions. In this study, we found 3 forms of insertion, 8 forms of deletion and 3 forms of substitution. The substitution model is seen in the form of a nucleotide replacement (SNP). The following is analysis for the pattern of mutations of MPB64 M. tuberculosis gene from 20 samples.

Table 4. Mutation pattern of DNA in MPB64 M. tuberculosis from 20 samples

\begin{tabular}{lllll}
\hline Type of Mutation & Position & $\mathrm{n}$ & $\%$ & Samples \\
\hline Insertions & $302 \mathrm{~A}$ & 2 & 10.0 & 4,12 \\
& $653 \mathrm{~T}$ & 14 & 70.0 & $1,5,6,7,8,9,10,11,13$, \\
& & & & $15,17,18,19,20$ \\
& $653 \mathrm{~A}$ & 2 & 10.0 & 4,12 \\
\hline Deletion & $647 \mathrm{~A}$ & 7 & 35.0 & $1,4,6,10,12,17,19$ \\
& $299-301$ & 1 & 5.0 & 8 \\
& 303 & 1 & 5.0 & 8 \\
& $309-311$ & 1 & 5.0 & 8 \\
\hline Substitution & $305 \mathrm{C}>\mathrm{A}$ & 1 & 5.0 & 8 \\
& $653 \mathrm{G}>\mathrm{T}$ & 6 & 30.0 & $2,3,4,12,14,16$ \\
& $651 \mathrm{~T}>\mathrm{G}$ & 6 & 30.0 & $2,3,4,12,14,16$ \\
\hline
\end{tabular}

*) Analysis using MPB64 H37Rv as a reference

Table 4 shows the pattern of DNA mutations of MPB64 M. tuberculosis genes in the form of insertion, deletion, and substitution wherein the highest form of insert mutation is $70 \%$, the highest delineation form $35 \%$ and the highest $30 \%$ substitution are found in turns of $653 \mathrm{G}>\mathrm{T}$ and 651T> G nucleotides. The MPB64 M. tuberculosis conserved genes lie in position 310 360.

For the design of the MBP64 conserved genes at the position of codon $310-360$, the sequence of MPB64 M. tuberculosis conserved gene to be used for primer design was obtained from the NCBI (National Centre for Biotechnology Information). First, we obtained properties or significant primer characteristics of design optimisation. Some of the primer characteristics were obtained a good quality of primer length, melting temperature (Tm), GC content, and molecular weight.

Based on the design result, the specific primer of MPB64 M. tuberculosis was shown in Table 5.

Table 5. Results of the specific design for MPB64 M. tuberculosis

\begin{tabular}{|c|c|c|c|c|c|c|c|c|c|}
\hline \multirow[b]{2}{*}{ Sequence } & \multicolumn{6}{|c|}{ Characteristic } & \multicolumn{3}{|l|}{$\mathrm{SSC}$} \\
\hline & $\begin{array}{l}\text { TM } \\
(\mathrm{oC})\end{array}$ & $\begin{array}{l}\mathrm{GC} \\
(\%)\end{array}$ & $\begin{array}{l}\text { MW } \\
\text { OD260 }\end{array}$ & $\begin{array}{l}\text { Mole } \\
\text { OD260 }\end{array}$ & $\begin{array}{l}\mu \mathrm{g} \\
\mathrm{OD} 260\end{array}$ & $\begin{array}{l}\text { ext.coef/L } \\
(\text { mole.cm) }\end{array}$ & $\mathrm{LF}$ & SF & DNA \\
\hline F_MPTAE2 & 53.2 & 45.0 & 6.079 & 5.0 & 30.3 & 200.700 & $(0.06) 25$ & 24 & 20 \\
\hline R_MPTAE2 & 55.2 & 50.0 & 6.157 & 5.1 & 31.4 & 196.200 & $(0.02) 25$ & 25.3 & 20 \\
\hline
\end{tabular}




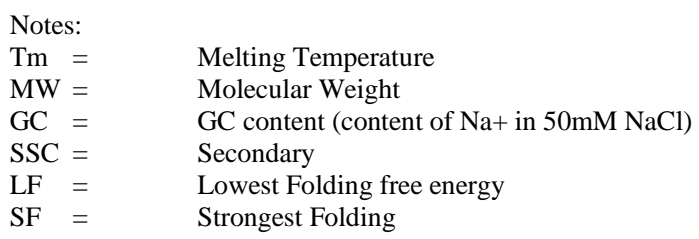

Table 5 shows the characteristics and number of oligonucleotides in both s of MPB64 (F_MPTAE2 and R_MPTAE2) were different, but the two primers were 20-bp.

\section{Discussions}

The screening test is an analytical method to determine the extent of a method can be used to diagnose a disease, which is considered its sensitivity and specificity values. Identification of bacteria M. tuberculosis is one of the parts in diagnosing TB which is not having satisfactory results. Diagnostics based on staining of acid-resistant bacilli using the Ziehl Neelsen method is the primer choice of early diagnosis of TB because it is practical, quick, and easy. This method is nonetheless less sensitive. Many studies have reported the sensitivity of the staining methods only $40-60 \%$ because it requires skilled workers in the process and the amount of sputum of at least 3-5 $\mathrm{ml}[16]$.

In conducting staining examinations in the laboratory, the examinees accidentally unrealised do a mistake in patients. The impact even causes major errors that affect the program [17]. Culturing of M. tuberculosis is rated as a standard of cause delayed treatment time for the patient.

As a result of some weaknesses of smear examination, some researchers developed a method of diagnosis of TB which is quick and easy but still has quality. Currently, the quick and easy but expensive examination that is being developed is the examination for TB cases. The sensitivity of the culture is much better than that of staining with only $10-100$ organisms per ml sample. Cultural methods are the gold standard of TB diagnosis but take a long time and PCR molecular test.

The success of the PCR process depends on the s used, and the good has a base number criterion of about 18-22 bp, more CG content, and optimum temperature.

In the design, it is known that GC content and dimer structure are an important part because GC content plays a role in improving stability. Strong hydrogen bonds in $\mathrm{G}$ and $\mathrm{C}$ base pairs cause more stable s attached to the template DNA, so GC content is recommended in the range of $40-60 \%$. In this study Table, 4.5 shows the GC content obtained in the sequence F_MPTAE2 as much as $45 \%$ and R_MPTAE2 as much as $50 \%$.

The annealing temperature (Ta) or the temperature of base repeating in the sequence is not used as a limitation in the study. The results of this study obtained melting temperature or melting temperature on sequence_F-MPTAE2 of 53.20C and R- MPTAE2 of 55.20C. An annealing temperature value that is proportional to the melting temperature value causes the annealing temperature not to be used as a characteristic considered in the design. Meanwhile, according to $\mathrm{Wu}$ et al. [18], it is advisable to avoid recurrence, because it can cause hairpin and road from primer to be not good, but very difficult to obtain without having hairpin structure. Hairpin at the 3 'delta $G$ end (the energy required to break the hairpin structure) $=-2 \mathrm{kcal} / \mathrm{mol}$ and an internal hairpin with $\mathrm{G}=-3 \mathrm{kcal} / \mathrm{mol}$ delta can still be tolerated. In this research, the delta $\mathrm{G}$ sequence F-MPTAE2 is $0.06 \mathrm{kcal} / \mathrm{mol}$ and sequence $\mathrm{R}$ - MPTAE2 $=0.02 \mathrm{kcal} / \mathrm{mol}$. Hairpin 
may be formed due to intra-molecular interaction in the resulting in folding of the 3 'end of the primer. The PCR reaction should not contain secondary structures in the form of hairpin or dimers.

The length of the oligonucleotides used as the optimum is about 18-22 nucleotides with GC content of about 50-60\%. Primer size that is too short and too long will cause mismatch primer and the PCR reaction is not effective, because the area attached to is not the desired area for amplification. The result of design in this research is $20 \mathrm{bp}$ which is not too short and not too long, so it is not expected to misprimer for diagnostic test of TB to DNA isolate in this dissertation research.

Some researchers consider the melting temperature as one of the characteristics to consider, such as Thermodynamic Basis Sets for Nearest Neighbor Interaction (TBSNNI) method to calculate the melting temperature. While there are other researchers, who use the method of Wallaces Formula [20].

In addition, some studies do not use the melting temperature as the design characteristics but replace it with melting temperature difference. The difference in melting temperature of a primer pair should not exceed $50^{\circ} \mathrm{C}$ in the hope that the pair is attached to the template at the same temperature [21].

The research related to the optimum design showing the required characteristics in performing using algorithmic methods to design the optimum PCR s. This study will be developed so that the specific design of MPB64 of M. tuberculosis for PCR modification in tuberculosis diagnostic testing.

\section{Conclusion}

Our study revealed that there are three types of mutations in MPB64 of M tuberculosis, namely insertion, deletion, and substitution. Conserved MPB64 M. tuberculosis lies along positions 310-360. The specific design of MPB64 M. tuberculosis consists of sequence codes F-MPTAE2 and R-MPTAE2 as diagnostic candidates for lung tuberculosis. We suggested that molecular diagnostic methods using the specific primer from conserved MPB64 M. tuberculosis is needed for highly sensitive and specific detection in patients for specific TB.

\section{References}

[1] World Health Organization: Global Tuberculosis Report. Tuberculosis Epidemiologi. 20th Edition. ISBN 97892: pp. 15 (2016)

[2] Zhu, C., Liu, J., Ling, Y., Yang, H., Liu, Z., Zheng, R., Qin, L., Hu, Y.: Evaluation of the Clinical Value of Elisa Based on MPT64 Antibody Aptamer for Serological Diagnosis of Pulmonary Tuberculosis. Bio Medical Central Infectious Diseases. Vol 12, pp. 96 (2012)..

[3] Burhan E. MDR TB. Pertemuan Ilmiah Pusat Studi TB HIV Unand di Padang. (2014)

[4] Susanty, E., Amir, Z., Siagian, P., Yunita, R., Eyanoer, PC.: Diagnostic test of GeneXpert MDR TB/RIF in General Hospital of Hajj Adam Malik, Medan. Jurnal Biosins. Vol 1 No 2: pp 19. (2015)

[5] Ministry of Health of Indonesia. Management of TB. Jakarta: Indonesian Ministry of Health. (2017).

[6] Cole, E., Cook, C. Characterization of infectious aerosols in health care facilities: an aid to effective engineering controls and preventive strategies". Am J Infect Control. Vol 26, pp 45364. (1998). 
[7] Hirano, K., Aono, A., Takahashi, M., Abe, C.: Polymorphism of Antigen MPT64 in MycobacteriuM. tuberculosis Strains. Journal of Clinical Microbiology.Vol. 42, pp. 390-92. (2004).

[8] Lee, JA., Tan, SC., Wang, CB., Tan, HK., Yap, PS., Low, JN., Chia.: Comparison of Protocols Involving Three Mycobacterial DNA Sequences, IS6110, 65 kDA Antigen and MPB64.J Neurol. Sei. Vol 123, pp 173-79. (1994)

[9] Liu, PYF., Shi, ZY., Lau, YJ., Hu, BS.: Rapid Diagnosis of Tuberkulosis Meningitis; a Simplofied Nested Amplification. Protocol . Neurology: pp 44 - 1161. (1994)

[10] Mustafa, AS.: HLA Promiscous Th1-cell Reactivity of MPB64 a Mayor Secreted Antigen of Mycobacterium tuberculosis in Healthy Subjects. Med. Princ. Pract. Vol 18, pp 385-92. (2009)

[11] Mustafa, H.: In Silico Binding Predictions for Identification of HLA DR Promiscuous Regions and Epitopes of Mycobacterium tuberculosis Protein MPT64 and their Recoqnition by Human Thi-cell. Med. Princ. Pract. 19: pp. 367-372. (2010)

[12] Mustafa, AS., Shaban, F.: Mapping of Th1-cell Epitope Regions of Mycobacterium. tuberculosis Protein MPB64 Using Synthetic Peptide and T-cell Lines from Mycobacterium. tuberculosis Infected Healthy Human. Med. Princ. Pract. Vol 19, pp. 122-28. (2010)

[13] Khosla, R., Dwivedi, A., Sarin, BC.,Sehajpal, PK.: Peripheral Blood Based C-PCR Assay for Diagnosing Extra-Pulmonary Tuberculosis. Indian Journal Experimental Biology. Vol 47, pp. 447-53. (2009)

[14] Parekh, KM., Inamdar, V., Jog, A., Kar, A.: A Comparative Study of the Diagnosis of Pulmonary Tuberculosis Using Convensional Tools and Polymerase Chain Reactions. Indian Journal of Tuberculosis. Vol 53, pp. 69-76. (2006)

[15] Kusum, S., Manish, M., Kapel, G., Aman, S., Pallab, R., Kumar, SS., Sudesh, P., Meera, S.: Evaluasi of PCR Using MPB64 s for Rapid Diagnosis Tuberculosis Meningitis. Open Access Scientific Reports. Vol 1( 3). (2012)

[16] Narotam, S., Veena, S., Chandra, NS., Raj, SP., Kushanha, RS., Skivani, S., Shayon, G., Amer, N., Singh, RK..: Conventional PCR Usege for the Detection of Mycobacterium. tuberculosis complex in Cerebrospinal Fluid by MPB64 Target PCR. International Journal of Drug Development and Research. pp. 206 - 7. (2012)

[17] Rezeki, M., Ida Parwati., Hernowo, S., Tjandrawati, A.: Validity Multiplex of Real Time Polymerase Chain Reaction for Limphadenitis Tuberculosis Diagnosis in Specimen Paraffin Block. Magazine of Medicine, Bandung. pp. 162-3. (2014)

[18] Girsang, M.: The errors in Sputum Examination of acid resistant bacilli in TB TB prevention program to several examination and identification of TB. Research and Development of Health Media. Vol.9 No.3. (1999)

[19] Wu, J.S., Lee, C.C., Shiue, Y.L.; design Using genetic Algorithm. Bioimformatics. pp. 1710 7. (2004)

[20] Yang, C.H., Cheng, Y.H., Chang, H.W., Chuang, L.Y.: Design with Spesific PCR Product Size Using Memetic Algorithm. IEEE Conf. Soft Comput Ind. Appl: pp. 332-7. (2008)

[21] Amoozegar, M., Reznannejad, E.: Design Using Gravitational search Algorithm. Iran. Conf. Intell. Syst:. pp. 1-6. (2014) 